\title{
1914 : neutralités, neutralismes en question
}

\section{3-14 NOVEMBRE 2014}

Alors que l'Europe s'embrase en août 1914 sans que personne n'imagine encore l'ampleur du conflit, les débats sont déjà nombreux sur la pertinence de l'engagement qu'il soit politique, idéologique, économique ou religieux. Les États, les groupes d'opinion et les individus optent pour des positions dictées par leur conscience ou par les intérêts politiques.

La guerre durant, les neutralismes les

\section{Intervenants :}

Anne Banny, Doyenne de la Faculté des

Lettres, ICP

Ineke Bockting, Professeur, ICP

Juan Manuel Bonet, Directeur de l'Instituto

Cervantes de Paris

Xavier Boniface, Professeur, Université de

Picardie Jules Verne

Javier Zamora Bonilla, Maître de Conférences,

Université Complutense de Madrid

Béatrice Fonck, Professeur, ICP

Jérôme Grondeux, Maître de Conférences,

Université Paris IV

Deborah Jenner, Docteur, Chargée de cours,

ICP, American Business School

Jennifer Kilgore-Caradec, Maître de

Conférences, Université de Caen

Henri Legohérel, Recteur honoraire et

Président, Académie de marine

Frédéric Le Moal, Enseignant, lycée militaire

de Saint-Cyr et Institut Albert le Grand

\section{Infos pratiques}

Institut Catholique de Paris 21 rue d'Assas Paris $6^{\mathrm{e}}$

Instituto Cervantes de Paris 7 Rue Quentin-Bauchart Paris 8 e www. paris.cervantes.es HDR, ICP Université Paris III plus affirmés vont être mis à mal. Dans le cadre d'une approche historique, géographique, littéraire et artistique. l'objet de ce colloque est d'examiner evolution des enjeux géopolitiques et des débats d'ordre moral, spirituel ou idéologique qui ont conduit les défenseurs de la neutralité à maintenir leur position pendant toutes ces années ou à s'engouffrer de gré ou de force dans la voie de l'interventionnisme.

Elli Lemonidou Professeur assistant, Université de Patras (Grèce) Emmanuel Lincot, Maître de conférences

Jean-Pierre Lozato-Giotart Professeur honoraire, Université Paris III Linda Martz, Maître de conférences,

Université Américaine de Paris Benoît Pellistrandi, Docteur, Professeur de Première supérieure, Lycée Condorcet Laura Pettinaroli, Maître de conférences, ICP Pauline Piettre, Maître de conférences, ICP Jean-Claude Rabaté, Professeur émérite,

Colette Rabaté, Maître de conférences honoraire, Université de Tours

Pierre Sicard, Professeur, Université de

Picardie, membre de l'Institut des Amériques

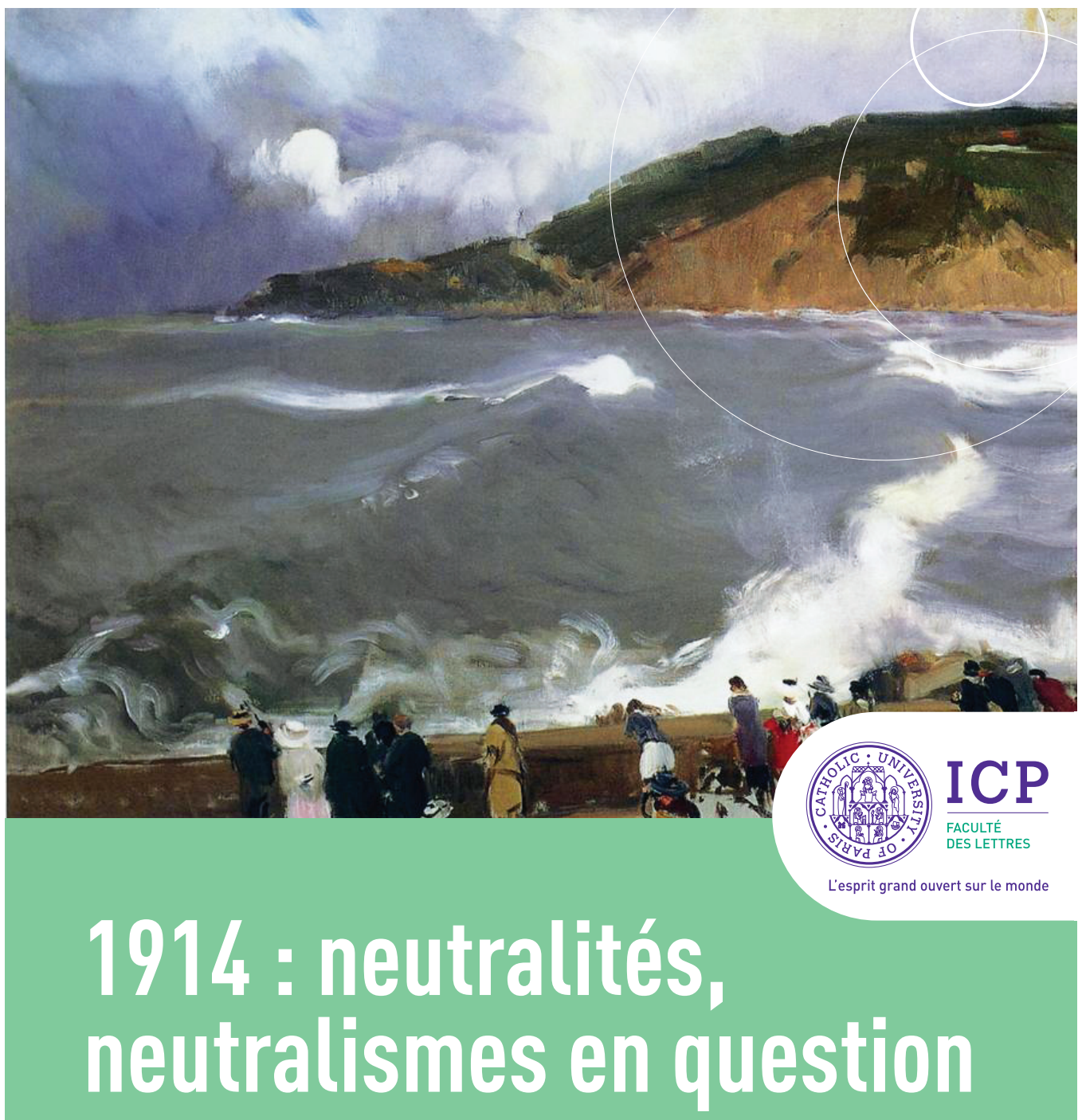

Colloque international et interdisciplinaire Organisation : I. Bockting, B. Fonck, P. Piettre de la Faculté des Lettres de I'ICP, en collaboration avec l'Instituto Cervantes de Paris et avec le soutien du Vice-Rectorat à la Recherche de l'ICP

13 \& 14 NOVEMBRE 2014

Entrée libre 


\section{Colloque international et interdisciplinaire}

\section{4 : neutralités, neutralismes en question}

\section{JEUDI 13 NOVEMBRE 2014}

À l'ICP, 21 rue d'Assas Paris 6

\section{9h30}

Ouverture par $\mathrm{M}^{\mathrm{me}}$ Anne Banny et

M. Juan Manuel Bonet

Atelier 1:

La neutralité : enjeux stratégiques et

éthiques

Présidence : $\mathbf{M}^{\mathrm{me}}$ Pauline Piettre

\section{0 h00}

Plénière La neutralité, introduction historique M. Henri Legohérel

\section{1 h00}

La géographie des neutralités au cours de la Première Guerre mondiale

M. Jean-Pierre Lozato-Giotart

11h30-11h45 Pause

\section{$12 \mathrm{ho0}$}

Le Saint-Siège dans la Grande Guerre,

entre neutralité et impartialité

M. Xavier Boniface

12h30-13h00 Questions et discussion

Atelier 2:

Neutralité ou engagement : les enjeux d'un conflit mondial

Présidence : $\mathbf{M}^{\text {me }}$ Linda Martz

\section{$15 \mathrm{~h} 00$}

La neutralité italienne en 1914 : un choix définitif? M. Frédéric Le Moal 15h30

Between loyalty and betrayal: Neutrality and Neutralism of American Nurses

During the Great War $\mathbf{M}^{\mathrm{me}}$ Ineke Bockting

\section{$16 h 00$}

Une neutralité inflammable ou quand tous désiraient la Grèce en tant qu'alliée $M^{\text {me }}$ Elli Lemonidou

16h30-16h45 Pause

\section{$17 \mathrm{~h} 00$}

La France devant l'attitude de la GrandeBretagne pendant la crise de l'été 1914 les enjeux d'un engagement commun $\mathrm{M}^{\text {me }}$ Pauline Piettre

\section{7h30}

Entre impartialité et engagement : le Saint-Siège face à la pression des belligérants et à l'urgence humanitaire sur le front russe (1914-1918) $\mathrm{M}^{\mathrm{me}}$ Laura Pettinaroli

\section{$18 \mathrm{h00}$}

De la Grande Guerre au Mouvement du 4 mai 1919. Mémoires et enjeux

M. Emmanuel Lincot

18h30 Questions et discussion suivies d'un apéritif à $19 \mathrm{~h} 00$

20h00 - 21 h00

Concert à l'ICP à la mémoire du compositeur Enrique Granados, interprété par $\mathbf{M}^{\text {me }}$ Odile Courtois. au piano et $\mathbf{M}^{\mathrm{me}}$ Anne Bouvier, soprano
VENDREDI 14 NOVEMBRE 2014

Matin : à l'ICP

Atelier 1 :

Questionnements individuels et

mouvements d'opinion 1

Présidence : $\mathrm{M}^{\mathrm{me}}$ Ineke Bockting

9h30

Woodrow Wilson et l'électorat démocrate face au conflit européen, août 1914-avril 1917 M. Pierre Sicard

\section{$10 \mathrm{h00}$}

La Plume blanche : patriotisme, pacifisme et neutralité chez les

suffragettes britanniques

$M^{\text {me }}$ Linda Martz

10h30

Great Artists views of the Great War $\mathrm{M}^{\mathrm{me}}$ Deborah Jenner

$11 \mathrm{~h} 00$

Neutralité et exil artistique : le cas de

l'Espagne M. Juan Manuel Bonet

\section{1h30-11h45 Pause}

\section{$12 \mathrm{~h} 00$}

Les poètes face à la guerre de 1914 quand la neutralité semble impossible $\mathrm{M}^{\mathrm{me}}$ Jennifer Kilgore-Caradec

$12 \mathrm{~h} 30$

Péguy dans la marche à la guerre

M. Jerôme Grondeux

13h-13h30 Questions et discussion
Après-midi : à l'Instituto Cervantes 7 rue Quentin Bauchart, Paris $8^{e}$

Atelier 2:

Questionnements individuels et mouvements d'opinion 2

Présidence : M. Juan Manuel Bonet

\section{$16 \mathrm{~h} 00$}

Aux origines de l'exception espagnole

la neutralité et ses conséquences

M. Benoît Pellistrandi

\section{$16 \mathrm{~h} 30$}

Intelectuales españoles y europeos ante la gran guerra M. Javier Zamora Bonilla

17h-17h15 Pause

17 h30

L'antineutralisme de Miguel de Unamuno M. Jean-Claude Rabaté et $M^{\text {me }}$ Colette Rabaté

\section{$18 \mathrm{~h} 00$}

Neutralisme et esprit européen d'après J. Ortega y Gasset $\mathbf{M}^{\mathrm{me}}$ Béatrice Fonck

18h30-19h00 Questions et discussion

Clôture 